\title{
金属板材循环拉压加载中力学行为的演化
}

\author{
于高潮 1,2 赵 军 1,2 \\ (1. 先进锻压成形技术与科学教育部重点实验室(燕山大学) 秦皇岛 066004; \\ 2. 燕山大学机械工程学院 秦皇岛 066004)
}

\begin{abstract}
摘要: 在曲梁往复弯曲过程中, 曲梁上任意质点均发生循环拉压加载变形, 但目前对往复弯曲过程中的力学行为仍不明确。 为深入认识往复弯曲变形过程中宏观力学行为的演化规律, 采用对称恒应变幅控制方式进行低周次不同应变幅值条件下的小 应变循环拉压加载试验, 研究了 304 不锈钢和 ST12 冷轧板两种材料循环拉压加载条件下的宏观力学表现, 探究了弹性模量、 屈服应力和塑性模量随循环周次的变化规律和预应变对稳定滞后回线的影响。试验研究表明：304 不锈钢和 ST12 两种材料 均具有循环软化特性; 经循环拉压加载后, 两种材料的弹性模量、屈服应力和塑性模量发生明显改变, 而且拉压不具有一致 性; 循环拉压加载可湮灭预应变造成的材料力学性能初始差异, 使材料力学性能变得均匀一致, 也间接证明了往复弯曲可将 同层纤维力学性能均匀化, 为新本构关系的构建奠定了基础。
\end{abstract}

关键词: 金属板材; 循环拉压; 往复弯曲; 预应变; 力学行为; 循环软化

中图分类号: TG304

\section{Evolution of Mechanical Properties in Cyclic Tension-compression Loading on Sheet Metal}

\author{
YU Gaochao $^{1,2}$ ZHAO Jun ${ }^{1,2}$ \\ (1. Key Laboratory of Advanced Forging \& Stamping Technology and Science (Yanshan University) \\ of Ministry of Education, Qinhuangdao 066004; \\ 2. College of Mechanical Engineering, Yanshan University, Qinhuangdao 066004)
}

\begin{abstract}
In the process of reciprocating bending, any particle of the curved beam is subjected to cyclic tension-compression deformation. However, the mechanical behaviour of reciprocating bending is still not clear. In order to understand the evolution law of macroscopic mechanical behaviour in the process of reciprocating bending, the small strain cyclic tension-compression test of low cycle is carried out by the symmetric constant strain amplitude control model. The macroscopic performance on the 304 stainless steel and ST12 cold rolled plate under cyclic tension-compression loading is studied; the variation laws of elastic modulus, yield stress and plastic modulus with cycle times are investigated; the effect of prestrain is studied on the stable hysteresis loop. The experimental results show that 304 stainless steel and ST12 cold rolled plate are characterized by cyclic softening; after cyclic tension-compression loading, the elastic modulus, yield stress and plastic modulus of the two kinds of materials change obviously, and the tension and compression do not have consistency; cyclic tension-compression loading can annihilate the initial difference of mechanical properties caused by prestrain, and make the mechanical properties of materials become uniform. It is also indirectly proved that reciprocating bending can make the mechanical properties of the same layer of fibers be homogenized, which lays the foundation for the construction of the new constitutive relationship.
\end{abstract}

Key words: sheet metal; cyclic tension-compression; reciprocating bending; prestrain; mechanical behaviour; cyclic softening

\section{0 前言}

在辊式矫直和辊式矫圆工艺 ${ }^{[1-4]}$ 中, 被矫试件经 历了多次往复弯曲变形过程, 其往复弯曲次数一般

* 国家自然科学基金(51575473)、河北省钢铁联合研究基金(E2016203209) 和燕山大学博士基金(BL18024) 资助项目。20180326 收到初稿, 20180801 收到修改稿
在几次或十几次。往复弯曲可以湮灭初始曲率的差 异, 最终使曲率统一到同一方向、同一数值, 这一 原理称为往复弯曲统一曲率定理 ${ }^{[5]}$ 。在往复弯曲统 一曲率定理提出及验证过程中, 仅研究了弹复后曲 率与弯曲次数的关系, 未涉及往复弯曲过程中宏观 力学行为的演化规律。

在曲梁往复弯曲过程中, 曲梁上任意质点均发 生循环拉压加载变形。曲梁经历一个往复弯曲变形 
过程, 各质点就经历一个循环拉压加载周次。赵永 祥等 ${ }^{[6]}$ 揭示了不锈钢管道焊缝金属的循环应力一应 变响应存在很大分散性, 并引入 “概率循环应力一 应变曲线” 的表征方法。金永等 ${ }^{[7]}$ 分析了板材通过拉 深筋时的循环塑性变形机理, 考虑板材方向性、加 工硬化特性和包申格效应, 建立了基于 Hill 正交各 向异性屈服条件和 Ziegler 随动硬化法则的材料模 型。张庆玲等 ${ }^{[8]}$ 研究了 Q235 在小变形量循环载荷作 用下的应力应变曲线及特征, 并给出了相应的数学 模型。循环拉压加载变形过程涉及形变强化、 Bauschinger 效应 ${ }^{[9-10]}$ 和循环硬化 (软化) ${ }^{[11]}$ 等, 国内外 学者对循环拉压加载条件下的材料本构关系进行了 许多研究 ${ }^{[12-16]}$, 其主要研究焦点为应力应变滞后回线 的非线性数学描述, 对该条件下材料力学行为的演 化规律未进行详细探讨。YOSHIDA ${ }^{[13-14]}$ 研究了大应 变循环拉压加载条件下弹塑性行为, 并总结了弹性 模量的变化规律, 采用合适的函数对其进行了描述。

在往复弯曲变形过程中, 曲梁各微梁段 ${ }^{[17]}$ 的初 始曲率不同, 则不同纤维层上质点的预应变不同; 另外, 不同层纤维的变形量不同, 则各质点循环拉 压加载的应变幅值不同。所以, 本文拟采用对称恒 应变幅控制方式进行低周次不同应变幅值条件下的 小应变循环拉压加载试验, 研究稳定金属材料循环 拉压加载的宏观表现, 探究弹性模量、屈服应力和 塑性模量随循环周次的变化规律; 研究预应变对稳 定滞后回线的影响, 从而更深入地认识往复弯曲变 形过程中宏观力学行为的演化规律。

\section{1 循环拉压加载试验}

\section{1 试验材料}

选用厚度为 $3 \mathrm{~mm}$ 的 304 不锈钢板和 ST12 冷轧 板两种材料进行循环拉压加载试验, 其化学成分分 别如表 1、2 所示。

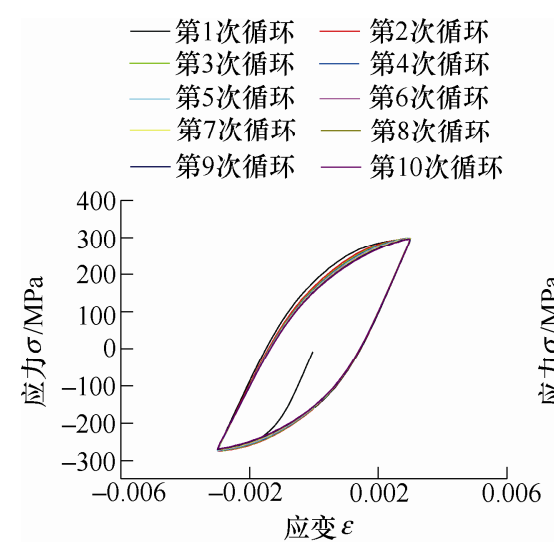

(a) $\Delta \varepsilon= \pm 0.003$

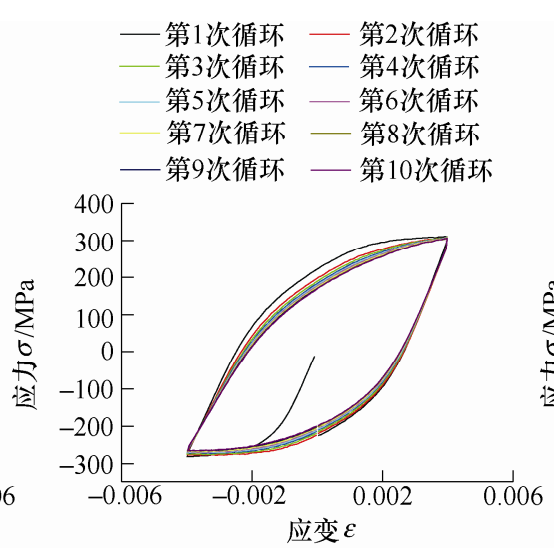

(b) $\Delta \varepsilon= \pm 0.004$

表 1 ST12 冷轧板化学成分(质量分数) \%

\begin{tabular}{ccccccc}
\hline \multirow{2}{*}{ 材料 } & $\mathrm{C}$ & $\mathrm{Si}$ & $\mathrm{Mn}$ & $\mathrm{P}$ & $\mathrm{S}$ & $\begin{array}{c}\text { Alt } \\
\text { Ni }\end{array}$ \\
& $\mathrm{Cr}$ & $\mathrm{Cu}$ & $\mathrm{As}$ & $\mathrm{N}$ & $\mathrm{Als}$ \\
\hline ST12 & 0.06 & 0.025 & 0.24 & 0.011 & 0.012 & 0.042 \\
\hline
\end{tabular}

表 2304 不锈钢化学成分(质量分数) \%

\begin{tabular}{cccccccc}
\hline 材料 & $\mathrm{C}$ & $\mathrm{Mn}$ & $\mathrm{P}$ & $\mathrm{S}$ & $\mathrm{Si}$ & $\mathrm{Cr}$ & $\mathrm{Ni}$ \\
\hline 304 & 0.08 & 2 & 0.045 & 0.03 & 1 & $18 \sim 20$ & $8 \sim 11$ \\
\hline
\end{tabular}

\section{2 试验设备与方法}

循环拉压加载试验在美国 MTS 公司生产的电液 伺服度劳试验机上进行, 其静励载荷量程为 $100 / 80 \mathrm{kN}$ 。

根据疲劳试验机参数要求和疲劳试验标准 ${ }^{[18]}$, 循 环拉压加载试件尺寸如图 1 所示, 试件的长度方向与 板材轧制方向平行。在往复弯曲过程中, 板坏的最大 应变一般可达到弹性极限应变的 5 倍左右 ${ }^{[3-5]}$, 且正 负应变的值可近似为相反数, 所以本文循环拉压加载 采用对称性恒应变幅控制模式, 应变幅值 $\Delta \varepsilon$ 分别选 用 $\pm 0.003 、 \pm 0.004$ 和 \pm 0.005 三组, 循环频率为 0.2 $\mathrm{Hz}$, 共循环拉压 10 周次, 这样保证了循环拉压加载 试验与往复弯曲中板材变形程度的一致性。

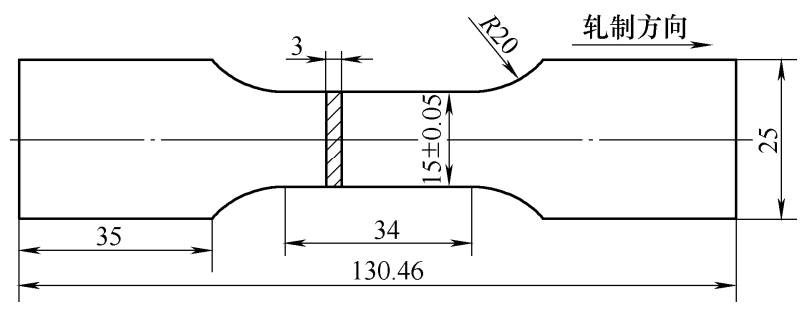

图 1 循环拉压加载试件尺寸

\section{2 循环拉压加载的宏观表现}

\section{1 滞后回线}

304不锈钢和 ST12 冷轧板两种材料的循环拉压 加载应力应变滞后回线分别如图 2、3 所示。图 4 为循环拉压加载后试件。

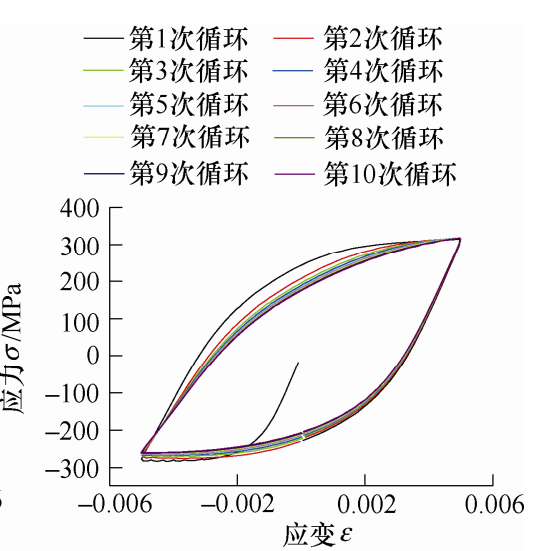

(c) $\Delta \varepsilon= \pm 0.005$

图 2304 不锈钢循环拉压加载应力应变滞后回线 


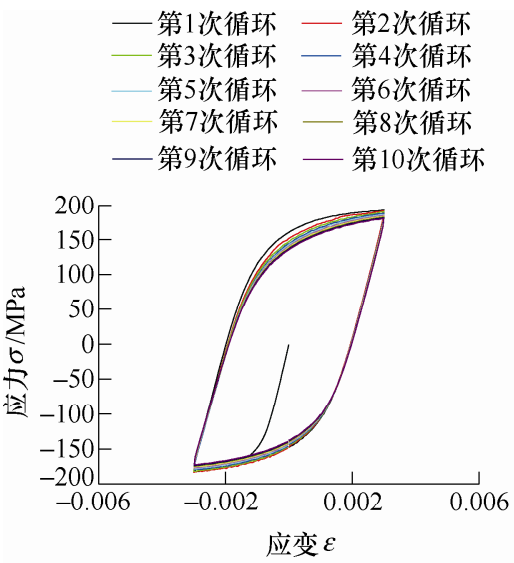

(a) $\Delta \varepsilon= \pm 0.003$

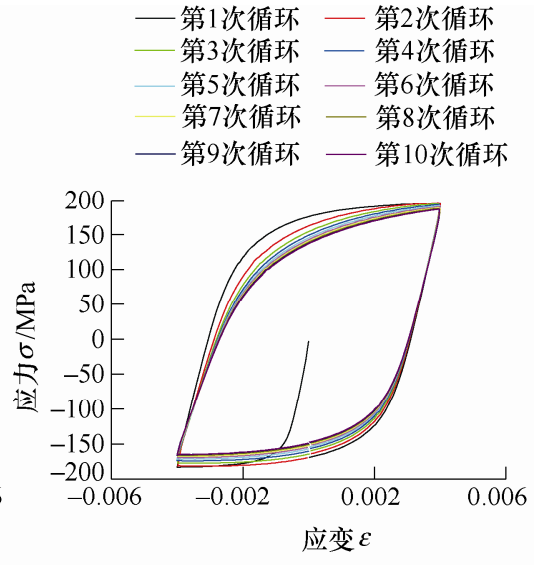

(b) $\Delta \varepsilon= \pm 0.004$

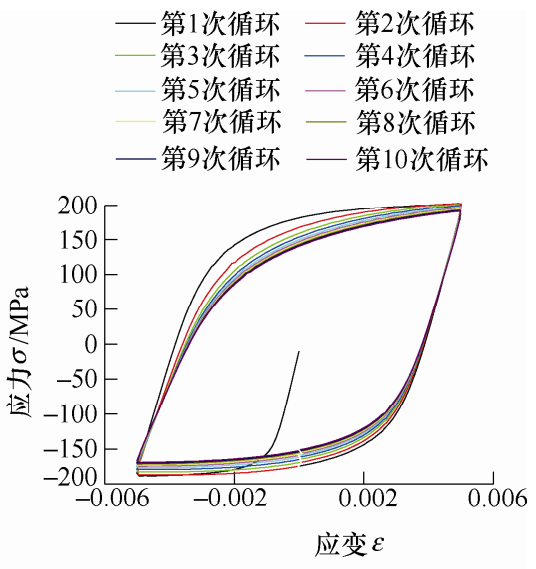

(c) $\Delta \varepsilon= \pm 0.005$

图 3 ST12 冷轧板循环拉压加载应力应变滞后回线

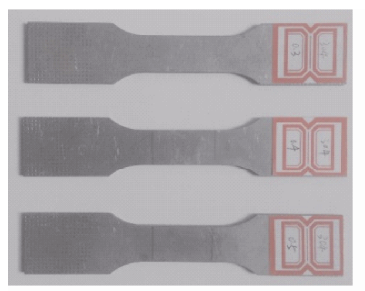

(a) 304 不锈钢

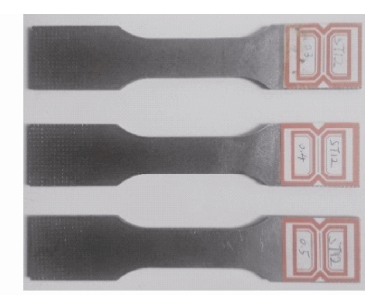

(b) ST12 冷轧板
图 4 循环拉压加载后试件

由图 2、3 可知, 304 不锈钢和 ST12 冷轧板试 件在恒应变幅低周次循环拉压加载过程中, 滞后回 线的包络面积随循环周次的增加逐渐减小, 且减小 量与应变幅值大小呈正相关, 两种材料明显发生了 循环软化现象。经 7 次左右循环周次, 滞后回线基 本稳定不变, 这说明了随循环周次的增加两种材料 的宏观力学性能逐渐趋于稳定。

图 5 为不同应变幅值下稳定应力应变滞后回 线、一次静拉与静压应力应变曲线及循环应力应变 曲线对比图。由图 5 可知, 在不同应变幅值情况下, 所得到的稳定应力应变滞后回线不同; 对于 304 不 锈钢, 在应变为正值塑性范围内, 循环应力应变曲 线和一次静拉与静压应力应变曲线基本重合。在应 变值为负值塑性范围内, 循环应力应变曲线明显高 于一次静拉与静压应力应变曲线, 表现为非对称循 环软化特性, 最大峰值应力的绝对值较最小峰值应 力的绝对值偏大 30 $60 \mathrm{MPa}$ 。同理, 对于 ST12 冷 轧板, 最大峰值应力的绝对值和最小峰值应力的绝 对值相差较小，近似属于对称循环软化。

\section{2 力学性能参数}

在理论解析和工程应用中, 研究人员或工程师 更关心循环加载对材料力学参数的影响, 以下研究 应变幅值 $\Delta \varepsilon= \pm 0.003$ 和 $\Delta \varepsilon= \pm 0.005$ 两种情况下循 环拉压加载对弹性模量、屈服应力和塑性模量的影 响规律。

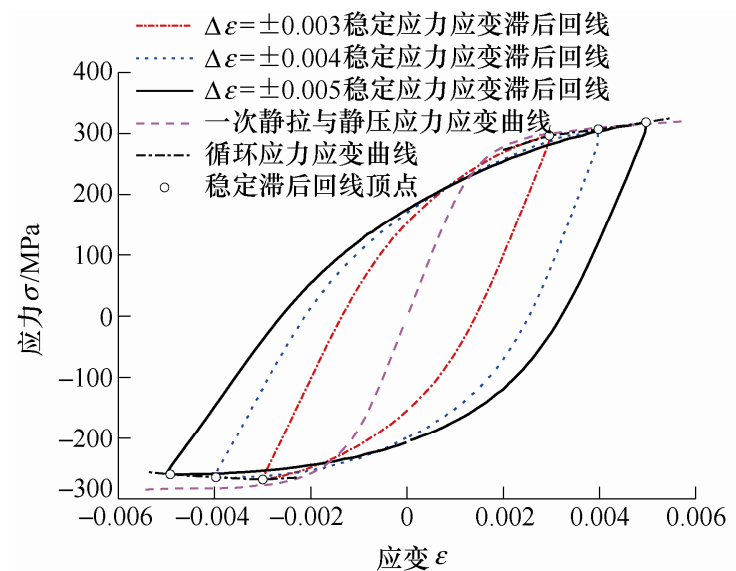

(a) 304 不锈钢

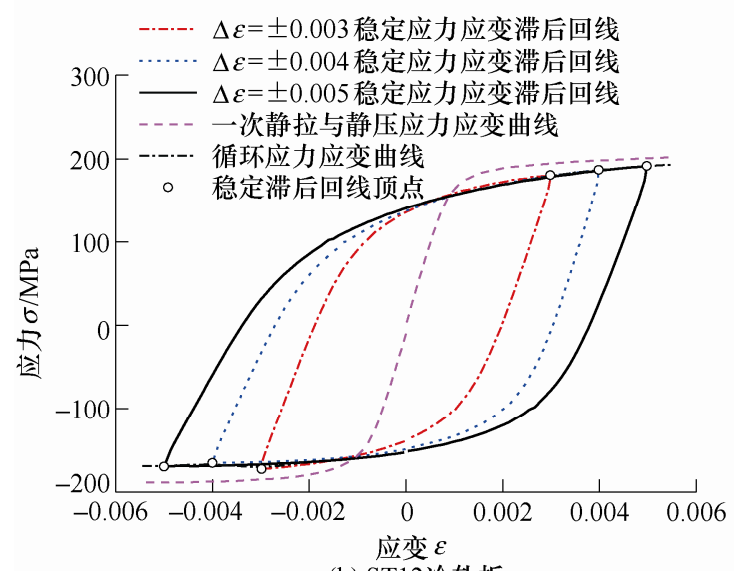

(b) ST12冷轧板

图 5 不同应变幅值下稳定应力应变滞后回线、一次静拉 与静压应力应变曲线和循环应力应变曲线

\subsection{1 弹性模量的变化规律}

将循环拉压加载过程中的弹性模量分为加载弹 性模量(LEM)和卸载弹性模量(UEM)。在弯曲弹复 预测中理论上应采用卸载弹性模量。然而, 在许 多弯曲弹复问题研究中将加载弹性模量近似为卸 载弹性模量, 因在一次弯曲弹复问题中可近似认 为拉压具有一致性，所以回弹预测值不会存在较 
大偏差 ${ }^{[17,19-20]}$ 。但在往复弯曲弹复问题中, 加载弹 性模量和卸载弹性模量均会随循环周次的增加而变 化, 所以循环加载过程中弹性模量的精细化研究对 往复弯曲弹复的精确预测与控制具有重要的意义。

一个循环拉压加载周期包括拉伸加载、拉伸卸 载、压缩加载和压缩卸载四个阶段, 故有四个弹性模 量, 包括拉伸加载弹性模量(TLEM)、拉伸卸载弹性 模量(CUEM)、压缩加载弹性模量(CLEM)和压缩卸载 弹性模量(CUEM)。因循环拉压为连续加载过程, 在 一个循环周次中假设拉伸加载弹性模量与压缩卸载 弹性模量相同, 压缩加载弹性模量与拉伸卸载弹性模 量相同, 故本节对循环拉压加载中拉伸卸载弹性模量 和压缩卸载弹性模量进行研究。对拉伸卸载部分和压 缩卸载部分数据点进行线性拟合, 从而得到 304 不锈 钢和 ST12 冷轧板循环拉压加载过程中卸载弹性模量 随循环周次的变化曲线，如图 6、7 所示。

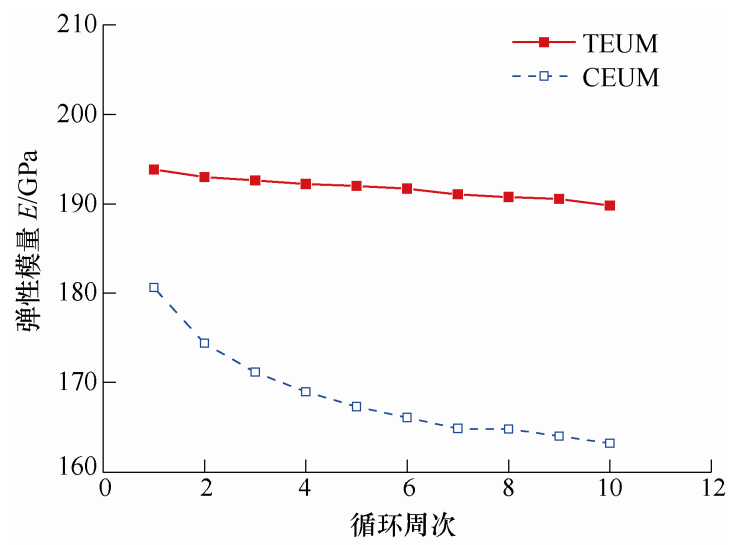

(a) $\Delta \varepsilon= \pm 0.003$

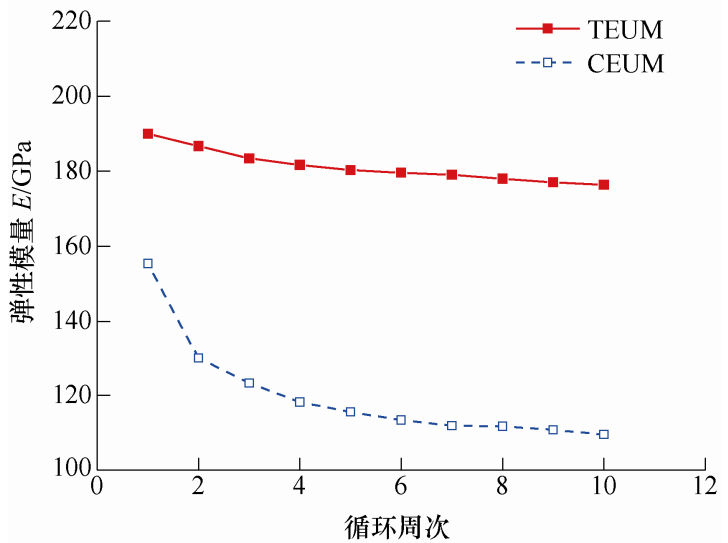

(b) $\Delta \varepsilon= \pm 0.005$

图 6304 不锈钢循环拉压加载过程中卸载弹性模量 随循环周次的变化曲线

由图 6、7 可知, 拉伸卸载弹性模量和压缩卸载 弹性模量均随循环周次的增加而减小, 最终两者的 值接近饱和。经循环拉压加载后, 两种材料的拉伸 卸载弹性模量和压缩卸载弹性模量均明显减小，而 且应变幅值越大, 拉伸卸载弹性模量和压缩卸载弹

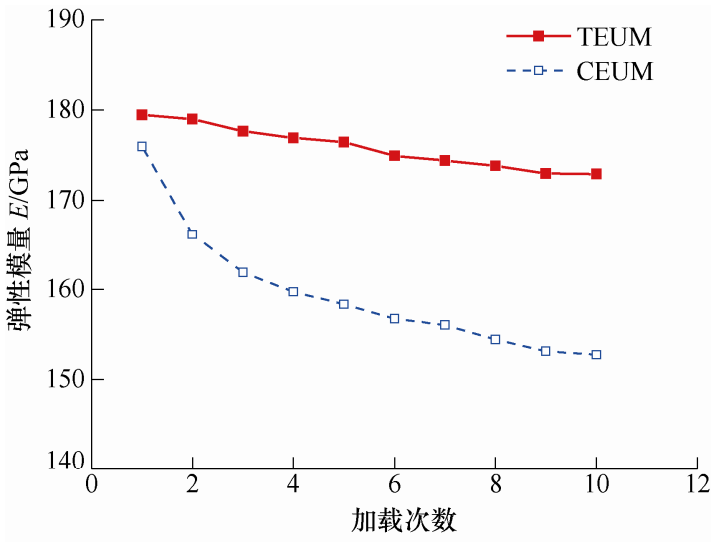

(a) $\Delta \varepsilon= \pm 0.003$

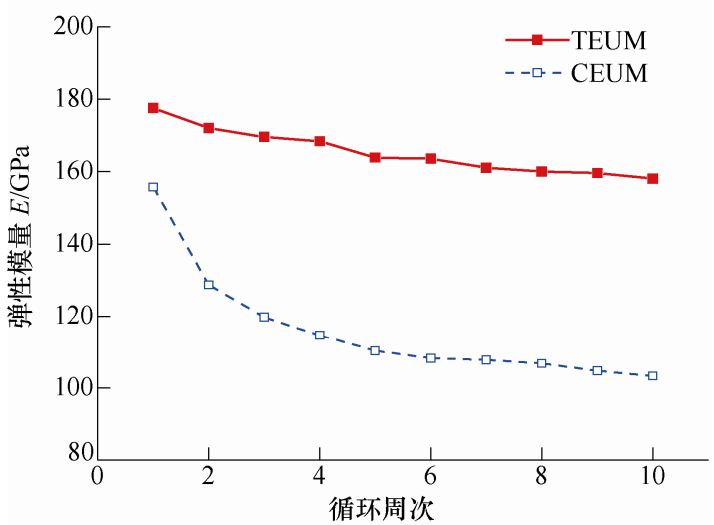

(b) $\Delta \varepsilon= \pm 0.005$

图 $7 \mathrm{ST} 12$ 冷轧板循环拉压加载过程中卸载弹性模量 随循环周次的变化曲线

性模量的减小量越大; 在相同应变幅值情况下，两 种材料的压缩卸载弹性模量减小量明显大于拉伸卸 载弹性模量减小量。在第 10 个循环周次, 弹性模量 已接近饱和, 但拉伸卸载弹性模量和压缩卸载弹性 模量的值存在明显差异。

\subsection{2 屈服应力的变化规律}

304 不锈钢和 ST12 冷轧板的滞后回线不存在明 显的屈服点，将总应变为 $0.2 \%$ 处的应力定义为屈服 应力。将循环拉压加载过程中的屈服应力分为拉伸 屈服应力(TYS)和压缩屈服应力(CYS)。304 不锈钢 和 ST12 冷车板循环拉压加载过程中屈服应力随循 环周次的变化曲线分别如图 8、9 所示。

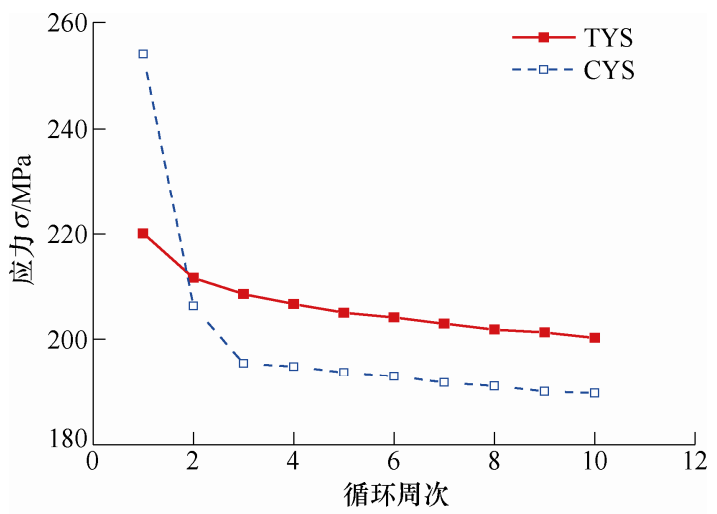

(a) $\Delta \varepsilon= \pm 0.003$ 


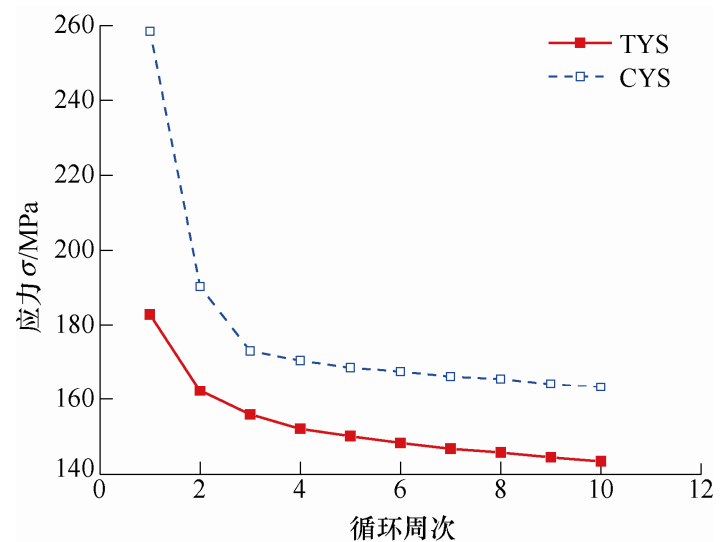

(b) $\Delta \varepsilon= \pm 0.005$

图 8304 不锈钢循环拉压加载过程中屈服应力 随循环周次的变化曲线

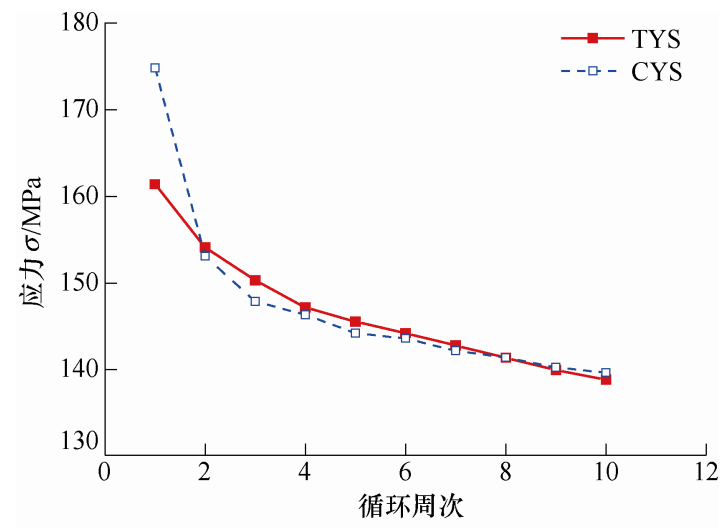

(a) $\Delta \varepsilon= \pm 0.003$

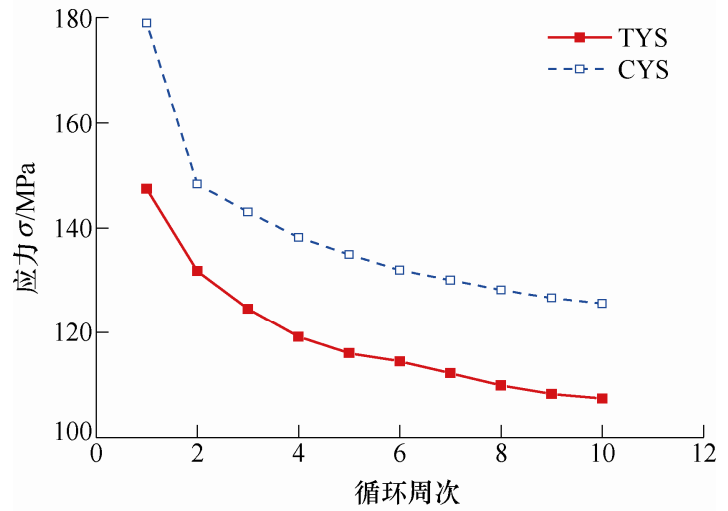

(a) $\Delta \varepsilon= \pm 0.005$

图 9 ST12 冷轧板循环拉压加载过程中屈服应力 随循环周次的变化曲线

由图 8、9 可知, 拉伸屈服应力和压缩屈服应力 随循环周次的变化规律同弹性模量类似, 也表现为 随循环周次的增加而减小, 最后两者的值接近饱和。 经循环拉压加载后, 两种材料的拉伸屈服应力和压 缩屈服应力均明显减小, 而且应变幅值越大, 拉伸 屈服应力和压缩屈服应力的减小量越大。在第 10 个循环周次, 屈服应力已接近饱和, 但拉伸屈服应力 和压缩屈服应力的值多数情况下会产生明显差异。

2.2.3 塑性模量的变化规律

将循环拉压加载过程中的塑性模量分为拉伸塑
性模量(TPM)和压缩塑性模量(CPM)。304 不锈钢和 ST12 冷轧板循环拉压加载过程中塑性模量随循环 周次的变化曲线分别如图 10、11 所示。

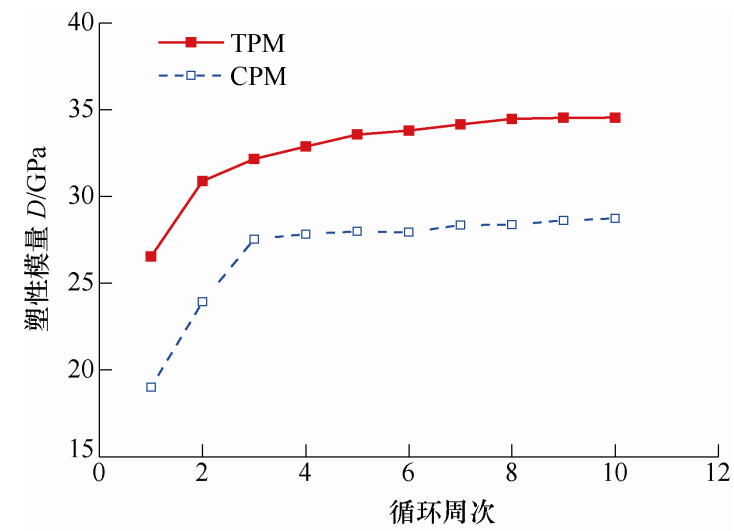

(a) $\Delta \varepsilon= \pm 0.003$

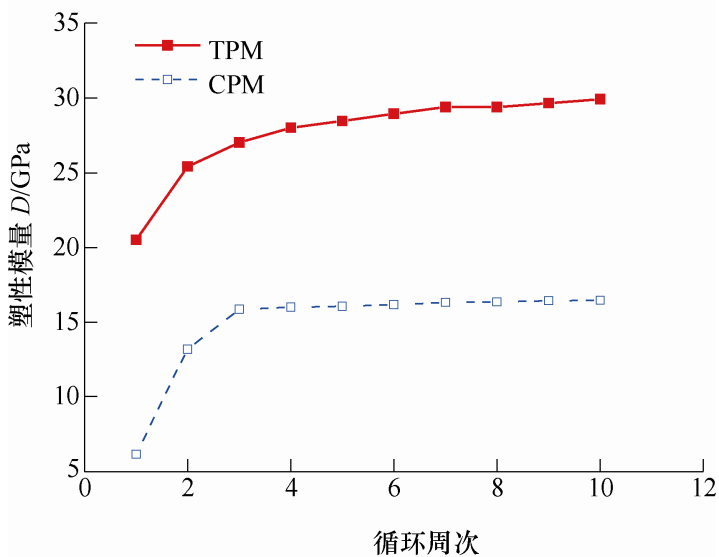

(b) $\Delta \varepsilon= \pm 0.005$

图 10304 不锈钢循环拉压加载过程中塑性模量 随循环周次的变化曲线

由图 10、11 可知, 304 不锈钢和 ST12 冷轧板 两种材料的拉伸塑性模量和压缩塑性模量均随循环 周次的增加而增大, 最终两者大小趋于稳定。经循 环拉压加载后，两种材料的拉伸塑性模量和压缩塑 性模量均明显增大，而且应变幅值越大，拉伸塑性 模量和压缩塑性模量的增大量越大。在第 10 个循环 周次, 塑性模量已趋于稳定, 但拉伸塑性模量和压 缩塑性模量的值存在明显差异。

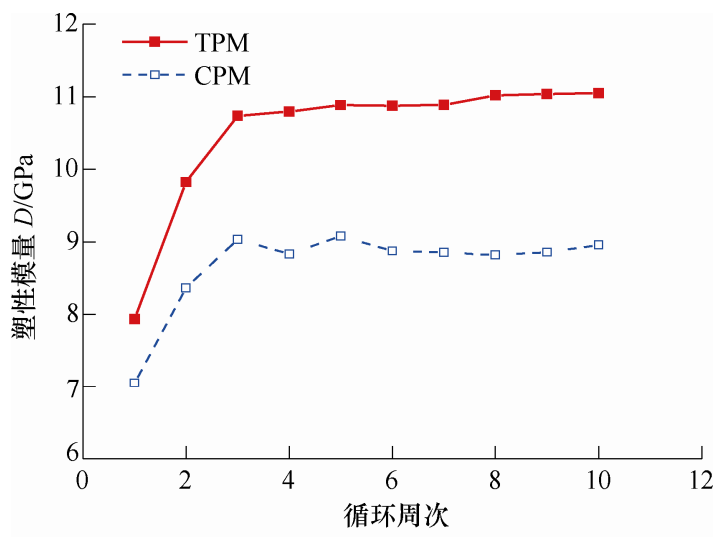

(a) $\Delta \varepsilon= \pm 0.003$ 


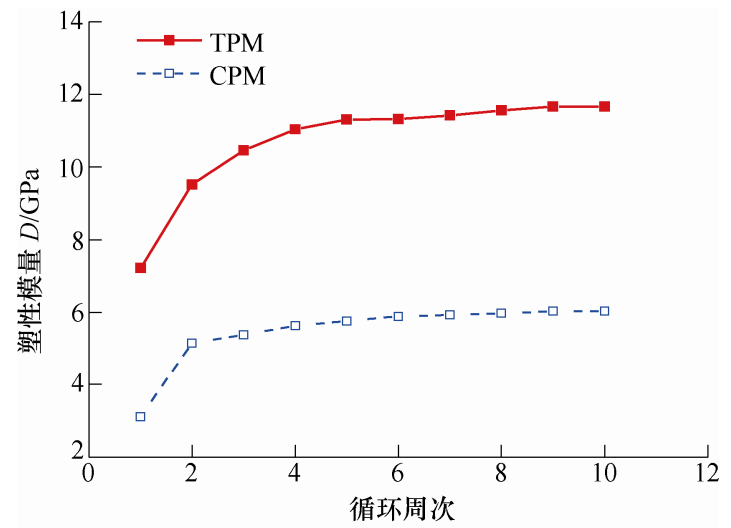

(b) $\Delta \varepsilon= \pm 0.005$

图 $11 \mathrm{ST} 12$ 冷轧板循环拉压加载过程中塑性模量 随循环周次的变化曲线

\section{3 预应变的影响}

在往复弯曲过程中, 初始待矫曲梁各质点的预应变 不同, 所以为了研究预应变在循环加载过程中对材料力 学性能的影响, 设计了不同预应变下的循环拉压加载试 验方案, 如表 3 所示。试验得到的 304 不锈钢和 ST12 冷轧板在 $\Delta \varepsilon= \pm 0.003$ 和 $\Delta \varepsilon= \pm 0.005$ 两种应变幅值不 同预应变下循环拉压加载滞后回线如图 12 15 所示。

\section{表 3 关于预应变的试验方案}

\begin{tabular}{ccc}
\hline 材料 & 应变幅值 $\Delta \varepsilon$ & 预应变 $\varepsilon_{\mathrm{p}}$ \\
\hline & & +0.005 \\
& & 0 \\
304 & \pm 0.003 & -0.005 \\
\cline { 2 - 3 } & & +0.003 \\
& \pm 0.005 & 0 \\
& & -0.003 \\
\hline \multirow{3}{*}{ ST12 } & \pm 0.003 & +0.005 \\
& & 0 \\
& & -0.005 \\
& & +0.003 \\
& & 0 \\
& & -0.003 \\
\hline
\end{tabular}

由图 12 15 可知, 304 不锈钢和 ST12 冷轧板在 不同预应变下循环拉压加载, 滞后回线的包络面积随 循环周次的增加逐渐减小, 说明每次循环加载所消耗 的塑性能逐渐减少，最后滞后回线趋于稳定，循环加 载所消耗的塑性能不变, 两种材料表现为循环软化, 这同无预应变下循环拉压加载具有相同的规律。为了 对比不同预应变、相同循环周次下滞后回线的差异， 选取第 1 循环周次、第 5 循环周次和第 10 循环周次的 滞后回线，对比研究其变化规律，如图 16 19 所示。

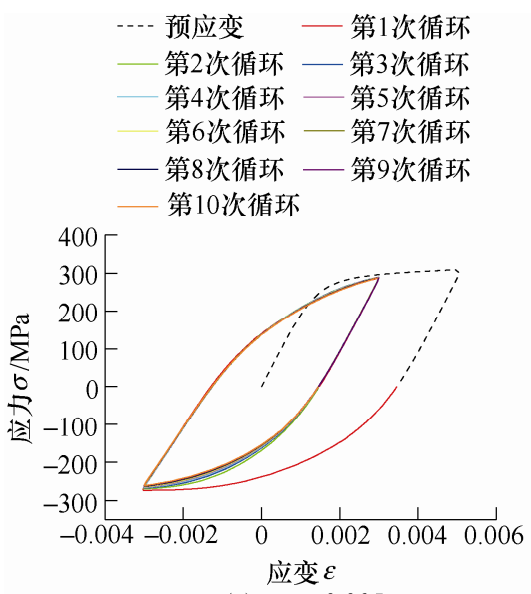

(a) $\varepsilon_{\mathrm{p}}=+0.005$

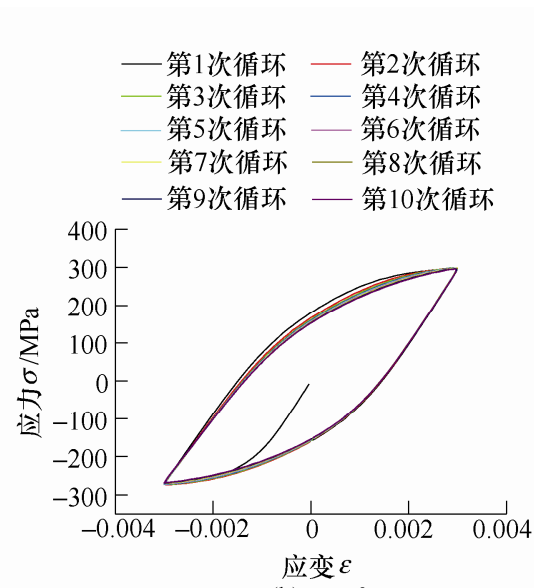

(b) $\varepsilon_{\mathrm{p}}=0$

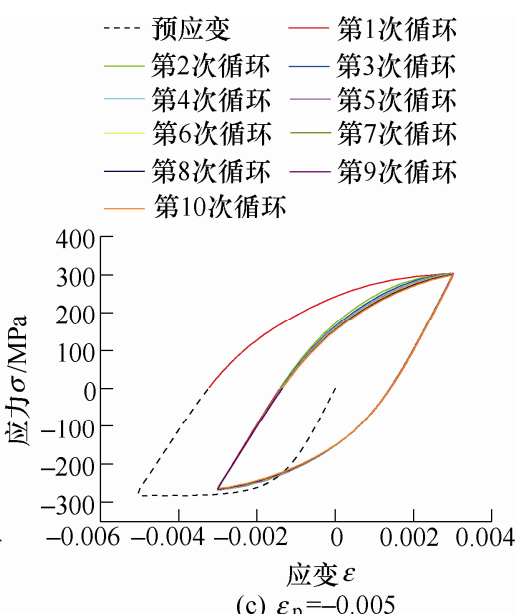

(c) $\varepsilon_{\mathrm{p}}=-0.005$

图 12 当 $\Delta \varepsilon= \pm 0.003$ 时不同预应变下 304 不锈钢循环拉压加载滞后回线

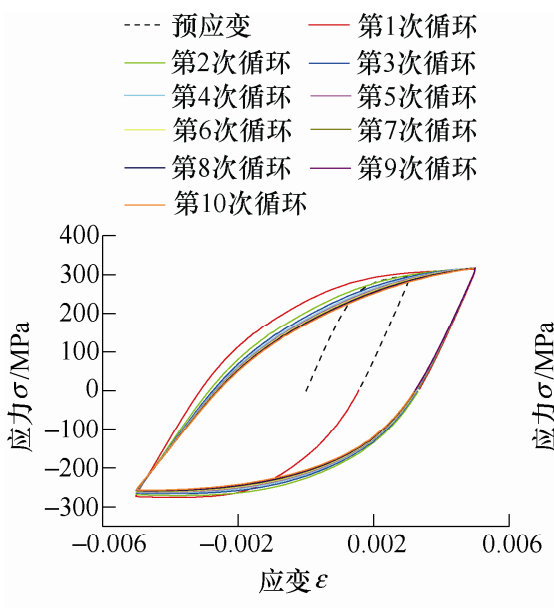

(a) $\varepsilon_{\mathrm{p}}=+0.005$

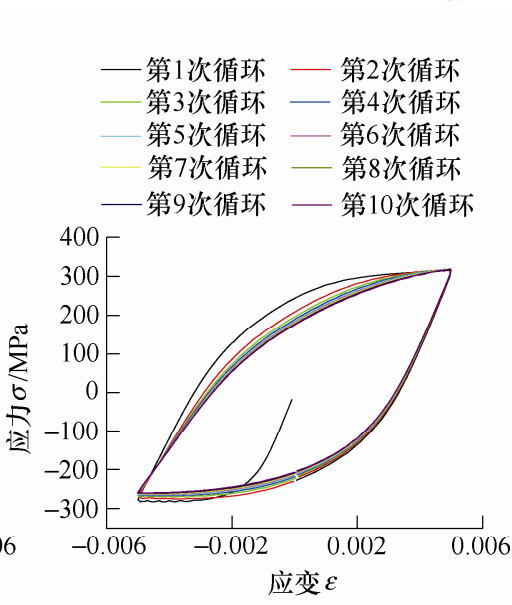

(b) $\varepsilon_{\mathrm{p}}=0$

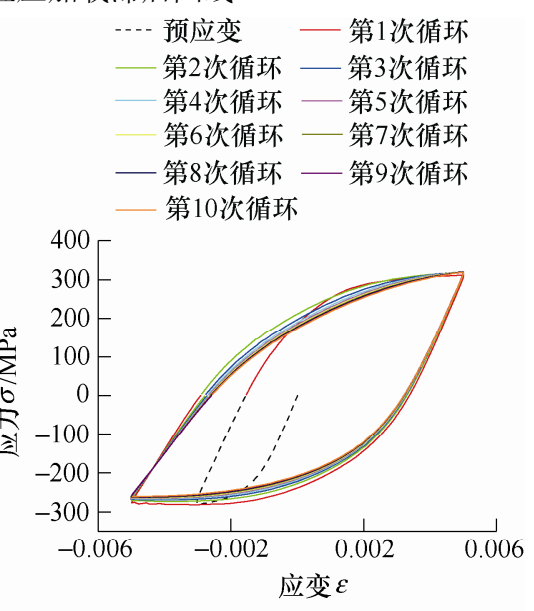

(c) $\varepsilon_{\mathrm{p}}=-0.005$

图 13 当 $\Delta \varepsilon= \pm 0.005$ 时不同预应变下 304 不锈钢循环拉压加载滞后回线 

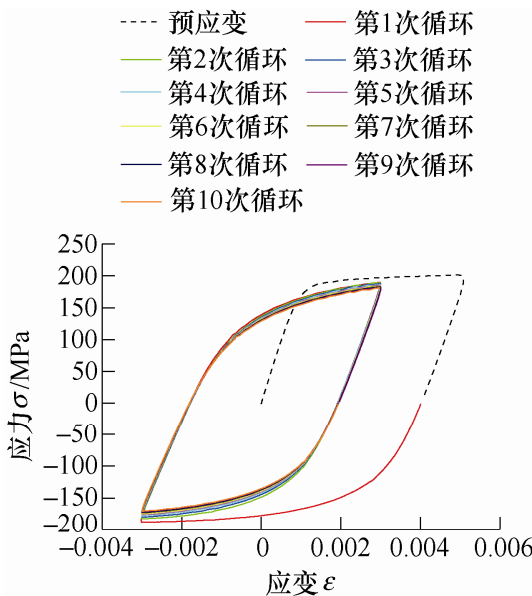

(a) $\varepsilon_{\mathrm{p}}=+0.005$

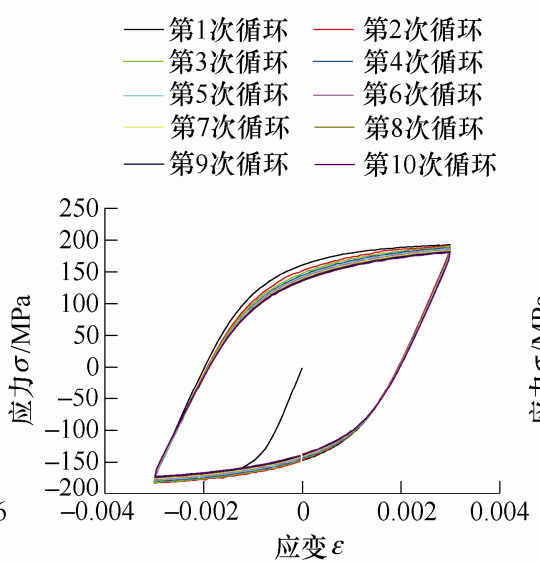

(b) $\varepsilon_{\mathrm{p}}=0$
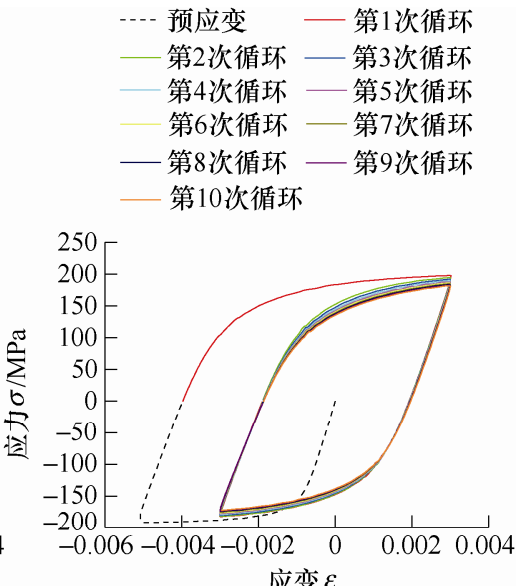

(c) $\varepsilon_{\mathrm{p}}=-0.005$

图 14 当 $\Delta \varepsilon= \pm 0.003$ 时不同预应变下 ST12 冷轧板循环拉压加载滞后回线

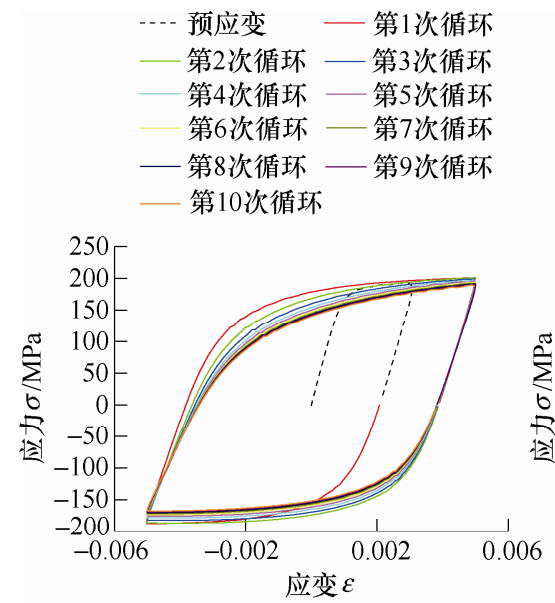

(a) $\varepsilon_{\mathrm{p}}=+0.005$

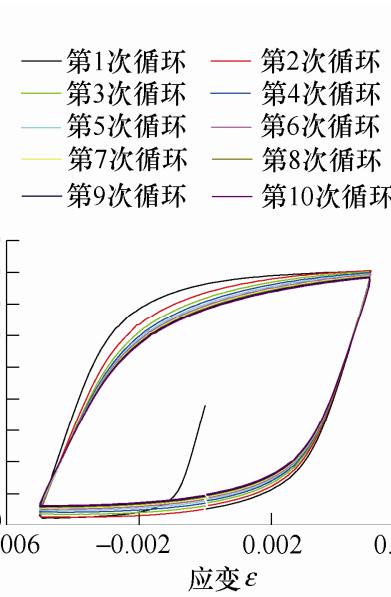

(b) $\varepsilon_{\mathrm{p}}=0$

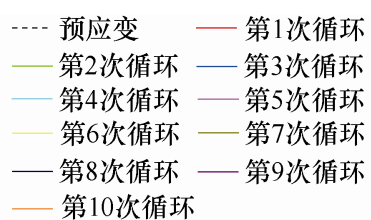

— 第10次循环

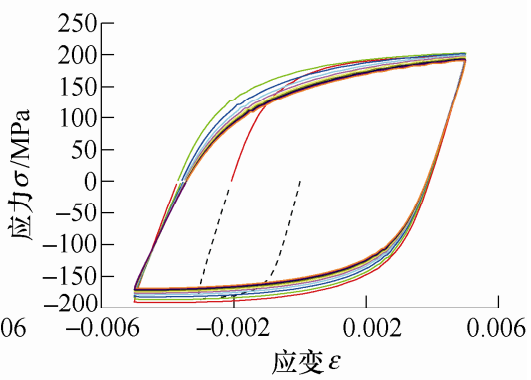

(c) $\varepsilon_{\mathrm{p}}=-0.005$

图 15 当 $\Delta \varepsilon= \pm 0.005$ 时不同预应变下 ST12 冷轧板循环拉压加载滞后回线

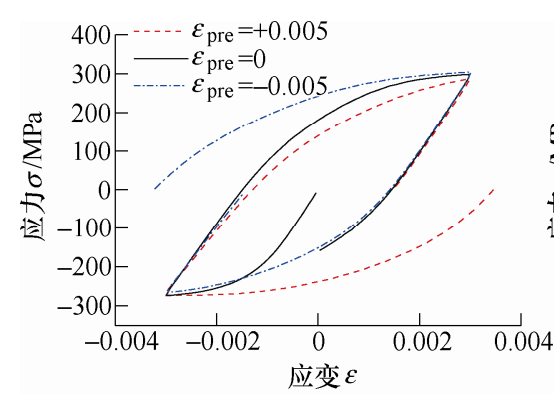

(a) 第1循环周次

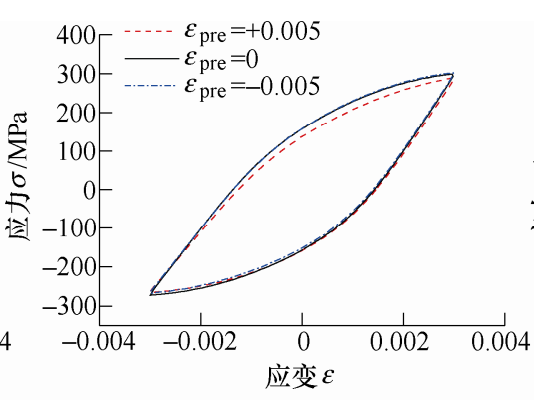

(b) 第5循环周次

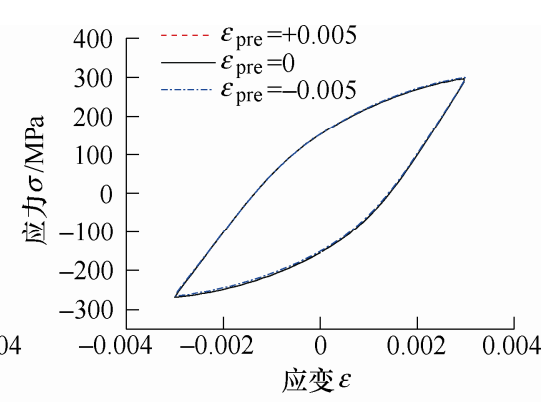

(c) 第10循环周次

图 16 当 $\Delta \varepsilon= \pm 0.003$ 时 304 不锈钢在不同预应变下滞后回线的对比

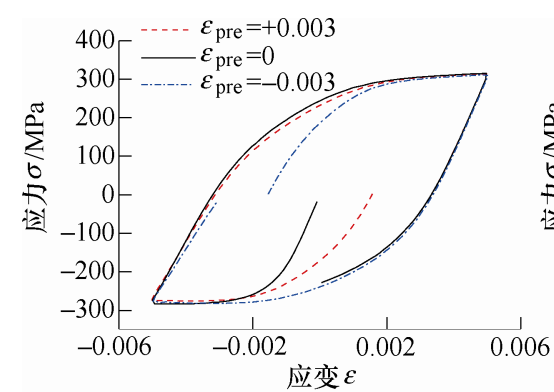

(a) 第1循环周次

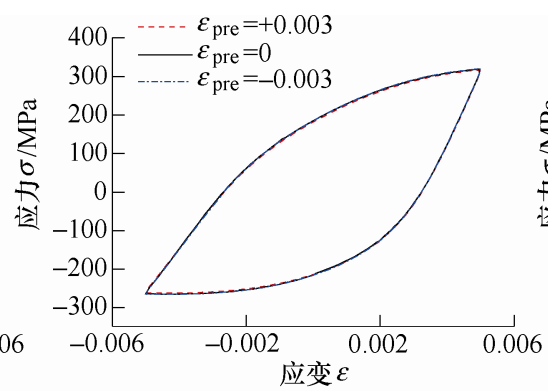

(b) 第5循环周次

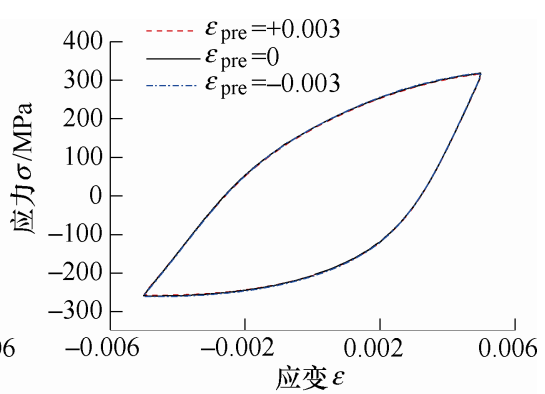

(c) 第10循环周次

图 17 当 $\Delta \varepsilon= \pm 0.005$ 时 304 不锈钢在不同预应变下滞后回线的对比 


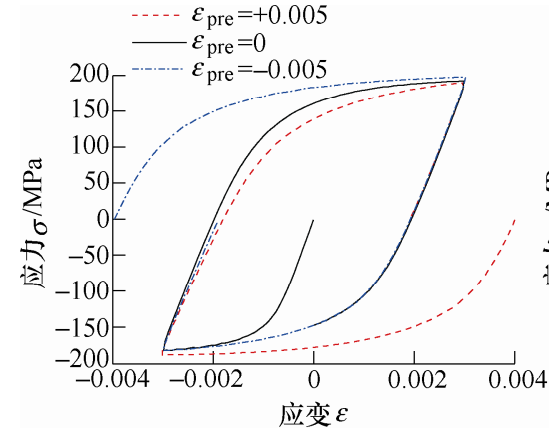

(a) 第1循环周次

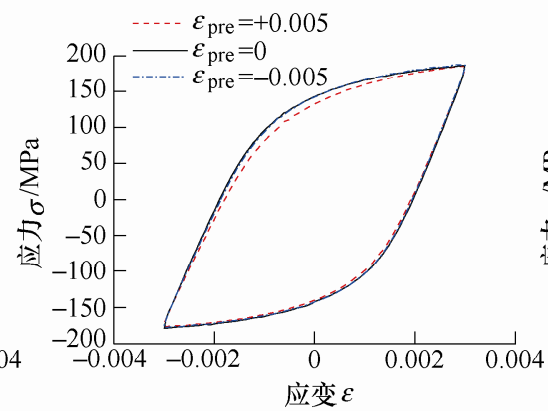

(b) 第5循环周次

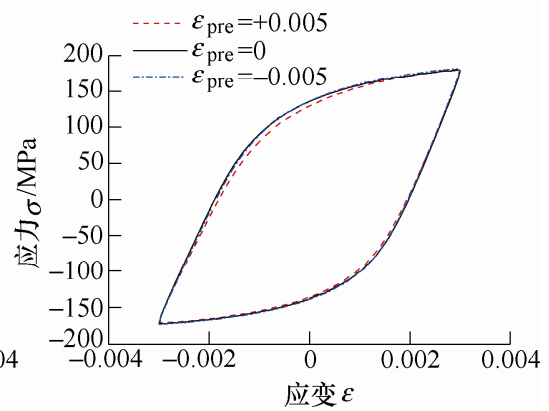

(c) 第10循环周次

图 18 当 $\Delta \varepsilon= \pm 0.003$ 时 ST12 冷轧板在不同预应变下滞后回线的对比

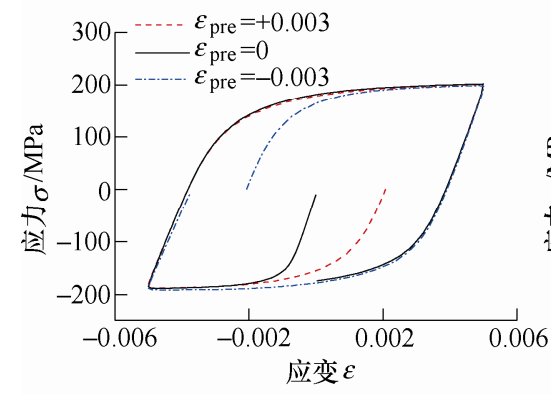

(a) 第 1 循环周次

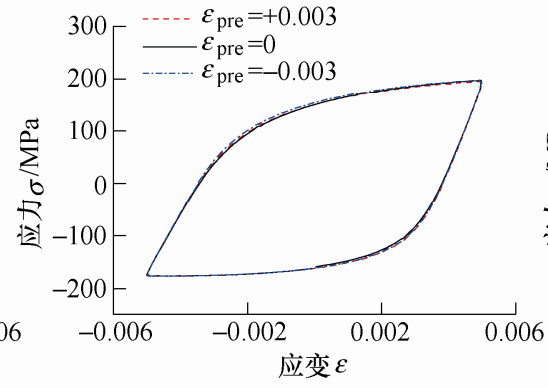

(b) 第5循环周次

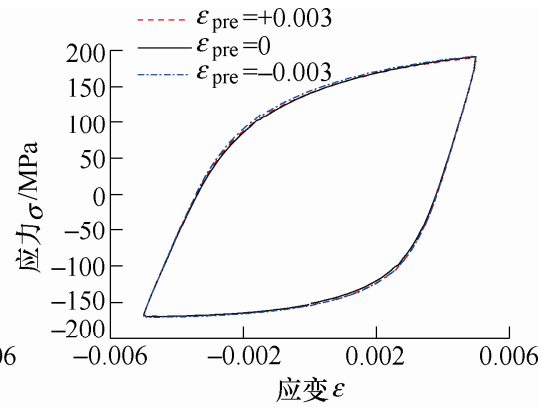

(c) 第10循环周次

图 19 当 $\Delta \varepsilon= \pm 0.005$ 时 ST12 冷轧板在不同预应变下滞后回线的对比

由图 16、18 可知, 304 不锈钢和 ST12 冷轧板 在应变幅值 $\Delta \varepsilon= \pm 0.003$ 循环拉压加载, 由于预应变 不同，第 1 循环周次三条不同预应变条件下的滞后 回线存在较大差异; 当加载到第 5 循环周次, 差异 明显减小; 最终加载到第 10 循环周次, 三条滞后回 线基本重合。同样，由图 17、19 可知，304 不锈钢 和 ST12 冷轧板在应变幅值 $\Delta \varepsilon= \pm 0.005$ 循环拉压加 载, 在第 5 循环周次, 三条滞后回线已基本重合。 这证明了预应变对稳定滞后回线的影响不大, 循环 拉压加载湮灭了预应变造成的材料力学性能的初始 差异, 使材料力学性能趋于均匀一致。这也间接证 明了往复弯曲不仅具有统一曲率的效果, 而且可将 同层纤维力学性能均匀化。

\section{4 结论}

(1) 304 不锈钢和 ST12 冷轧板两种材料均具有 循环软化特性。

(2) 经循环拉压加载后, 304 不锈钢和 ST12 冷 轧板两种材料不具有拉压一致性。其拉伸卸载弹性 模量与压缩卸载弹性模量和拉伸屈服应力与压缩屈 服应力的值不但明显减小，而且存在明显的差异; 拉伸塑性模量与压缩塑性模量的值不但明显增加, 而且存在明显的差异。所以, 在求解往复弯曲弹塑 性变形问题时, 需考虑材料的拉伸与压缩材料性能 的差异。
（3）在循环拉压加载过程中，预应变对稳定滞 后回线无明显影响。循环拉压加载可湮灭预应变造 成的材料力学性能初始差异, 使材料力学性能变得 均匀一致; 也间接证明了往复弯曲不仅具有统一曲 率的效果，而且可将同层纤维力学性能均匀化，更 深入地认识了往复弯曲过程中材料的宏观力学性能 演化规律。

(4) 在后续工作中仍需用弹塑性力学及材料学 相关理论对本构关系的构建及演化机理进行研究。

\section{参 考 文 献}

[1] 于高潮, 赵军, 邢娇娇, 等. 对称式三辊矫圆工艺研究 [J]. 机械工程学报，2017，53(14): 136-143.

YU Gaochao, ZHAO Jun, XING Jiaojiao, et al. Research on the symmetrical three-roller setting round process[J], Journal of Mechanical Engineering, 2017, 53(14) : 136-143.

[2] ZHAO Jun, YU Gaochao, MA Rui. A mechanical model of symmetrical three-roller setting round process: The static bending stage $[\mathrm{J}]$. Journal of Materials Processing Technology, 2016, 231: 501-512.

[3] YU Gaochao, ZHAO Jun, ZHAI Ruixue, et al. Theoretical analysis and experimental investigations on the symmetrical three-roller setting round process[J]. International Journal of Advanced Manufacturing Technology, 2018, 94: 45-56.

[4] YU Gaochao, ZHAI Ruixue, ZHAO Jun, et al. Theoretical 
analysis and numerical simulation on the process mechanism of two-roller straightening[J]. International Journal of Advanced Manufacturing Technology, 2018, 9(9-12): 4011-4021.

[5] 于高潮, 赵军, 马瑞, 等. 往复弯曲统一曲率定理及其 试验验证[J]. 机械工程学报, 2016, 18: 57-63.

YU Gaochao, ZHAO Jun, MA Rui, et al. The uniform curvature theorem by reciprocating bending and its experimental verification[J]. Journal of Mechanical Engineering, 2016, 18: 57-63.

[6] 赵永翔, 王金诺, 高庆. 概率循环应力一应变曲线及其 估计方法[J]. 机械工程学报, 2000，8：102-106.

ZHAO Yongxiang, WANG Jinnuo, GAO Qing. Probabilistic cyclic stress-strain curve and its estimation method[J]. Journal of Mechanical Engineering, 2000, 8: 102-106.

[7] 金沝, 李群, 蔡星周, 等. 板材通过拉深筋时的循环塑 性变形机理分析[J]. 机械工程学报, 2013, 49(12): 38-42.

JIN Miao, LI Qun, CAI Xingzhou, et al. Research on cyclic plastic deformation mechanism of sheet flowing through drawbead[J]. Journal of Mechanical Engineering, 2013, 49(12): 38-42.

[8] 张庆玲, 金沝, 张洪生, 等. 小变形循环载荷下 Q235 材料特性的试验研究[J]. 机械工程学报, 2017, 53(20): 69-76.

ZHANG Qingling, JIN Miao, ZHANG Hongsheng, et al. Experiment research on material characteristics of q235 under small deformation cyclic loading[J]. Journal of Mechanical Engineering, 2017, 53(20): 69-76.

[9] 张功庭, 盛光敏, 黄利. 金属包申格效应的表征、影响 因素与机理研究进展 [J]. 材料导报, 2008, 22(s3): 135-138.

ZHANG Gongting, SHENG Guangmin, HUANG Li. Research progress in characterization, influencing factors and mechanisms of bauschinger effect in metals material[J]. Materials Review, 2008, 22(s3): 135-138.

[10] ZANG Shunlai, LEE MG, LI Sun, et al. Measurement of the Bauschinger behavior of sheet metals by three-point bending springback test with pre-strained strips[J]. International Journal of Plasticity, 2014, 59(10): 84-107.
[11] MUTRUX A, BERISHA B, HORA P. Prediction of cyclic softening in a medium carbon steel during cross roll straightening[J]. Journal of Materials Processing Technology, 2011, 211(8): 1448-1456.

[12] ZHAO Kunmin. Cyclic stress-strain curve and springback simulation[D]. Ohio: The Ohio State University, 1999.

[13] YOSHIDA F. A constitutive model of cyclic plasticity[J]. International Journal of Plasticity, 2000, 16(3-4): 359-380.

[14] YOSHIDA F, UEMORI T, FUJIWARA K. Elastic-plastic behavior of steel sheets under in-plane cyclic tension-compression at large strain[J]. International Journal of Plasticity, 2002, 18: 633-659.

[15] KHUTIA N, DEY P, HASSAN T. An improved nonproportional cyclic plasticity model for multiaxial low-cycle fatigue and ratcheting responses of 304 stainless steel[J]. Mechanics of Materials, 2015, 91: 12-25.

[16] HU Fangxin, SHI Gang, SHI Yongjiu. Constitutive model for full-range elasto-plastic behavior of structural steels with yield plateau: Calibration and validation[J]. Engineering Structures, 2016, 118: 210-227.

[17] ZHAO Jun, YIN Jing, MA Lixia, et al. Springback equation of small curvature plane bending[J]. Science China: Technological Sciences, 2011, 54(9): 2386-2396.

[18] American Society of Testing Materials. Standard practice for strain-controlled fatigue testing[S]. West Conshohocken, United States, 1998.

[19] ZHAO Jun, ZHAN Peipei, MA Rui, et al. Quantitative prediction of reduction in large pipe setting round process[J]. Chinese Journal of Mechanical Engineering, 2013, 26(4): 722-729.

[20] ZHAO Jun, SONG Xiaokang. Control strategy of multi-point bending one-off straightening process for LSAW pipes[J]. International Journal of Advanced Manufacturing Technology, 2014, 72: 1615-1624.

作者简介: 于高潮, 男, 1988 年出生, 博士, 讲师。主要研究方向为板 管成形理论及新工艺和板材智能成形技术。

E-mail: ygc@ysu.edu.cn

赵军(通信作者), 男, 1957 年出生, 博士, 教授, 博士研究生导师。主 要从事板材成形及其智能化控制技术和精密塑性成形技术研究。

E-mail: zhaojun@ysu.edu.cn 